\title{
Hopf-type Estimates for Viscosity Solutions to Concave-Convex Hamilton-Jacobi Equations
}

\author{
NGUYEN DUY Thai Son \\ Kyoto Sangyo University \\ (Communicated by $\mathrm{H}$. Ishii)
}

\section{Introduction.}

Consider the Cauchy problem for the simplest Hamilton-Jacobi equation, namely,

$$
\begin{gathered}
\partial u / \partial t+f(\partial u / \partial x)=0 \quad \text { in } \quad \mathcal{D}:=\left\{t>0, x \in \mathbf{R}^{n}\right\}, \\
u(0, x)=\phi(x) \text { on }\left\{t=0, x \in \mathbf{R}^{n}\right\} .
\end{gathered}
$$

Here, $\partial / \partial x:=\left(\partial / \partial x_{1}, \cdots, \partial / \partial x_{n}\right)$. Let $\operatorname{Lip}(\overline{\mathcal{D}}):=\operatorname{Lip}(\mathcal{D}) \cap C(\overline{\mathcal{D}})$, where $\operatorname{Lip}(\mathcal{D})$ is the set of all locally Lipschitz continuous functions $u=u(t, x)$ defined on $\mathcal{D}$. A function $u \in$ $\operatorname{Lip}(\overline{\mathcal{D}})$ will be called a global solution of the Cauchy problem (1)-(2) if it satisfies (1) almost everywhere in $\mathcal{D}$, with $u(0, \cdot)=\phi$. A global solution of (1)-(2) is given by explicit formulas of Hopf [3] in the following two cases:

(a) $f$ convex (or concave) and $\phi$ largely arbitrary; and

(b) $\phi$ convex (or concave) and $f$ largely arbitrary.

It is unlikely that such restrictions, either on the Hamiltonian $f=f(p)$ or on the initial data $\phi=\phi(x)$, are really vital. A relevant solution is expected to exist under much wider assumptions. According to Hopf, that he has been unable to get further is doubtless due to a limitation in his approach: he uses the Legendre transformation globally, and this global theory has been carried through only in the case of convex (or concave) functions (Fenchel's theory of conjugate convex (or concave) functions).

We shall often suppose in this note that $n=n_{1}+n_{2}$ and that the variable $p \in \mathbf{R}^{n}$ is separated into two as $p=\left(p^{\prime}, p^{\prime \prime}\right)$ with $p^{\prime} \in \mathbf{R}^{n_{1}}, p^{\prime \prime} \in \mathbf{R}^{n_{2}}$. (Similarly for $x, z, \cdots \in$ $\mathbf{R}^{n}$. In particular, the zero-vector in $\mathbf{R}^{n}$ will be $0=\left(0^{\prime}, 0^{\prime \prime}\right)$, where $0^{\prime}$ and $0^{\prime \prime}$ stand for the zero-vectors in $\mathbf{R}^{n_{1}}$ and $\mathbf{R}^{n_{2}}$, respectively.) Recall (see Rockafellar [6]) that a function $f=f\left(p^{\prime}, p^{\prime \prime}\right)$ is called concave-convex if it is concave in $p^{\prime} \in \mathbf{R}^{n_{1}}$ for each $p^{\prime \prime} \in \mathbf{R}^{n_{2}}$ and convex in $p^{\prime \prime} \in \mathbf{R}^{n_{2}}$ for each $p^{\prime} \in \mathbf{R}^{n_{1}}$. We have proposed in [7] to examine a class of

Received February 14, 2000

Revised June 27, 2000

On leave from Hue University of Vietnam. Supported by the JSPS Postdoctoral Fellowship for Foreign Researchers in Japan. 
concave-convex functions as a more general framework where the discussion of the global Legendre transformation still makes sense. A Hopf-type formula for global solutions to nonconcave, non-convex Hamilton-Jacobi equations can thereby be considered.

If $f$ is a (concave-convex) function given by a special representation

$$
f\left(p^{\prime}, p^{\prime \prime}\right):=H_{1}\left(p^{\prime}\right)+H_{2}\left(p^{\prime \prime}\right) \text { on } \mathbf{R}^{n_{1}} \times \mathbf{R}^{n_{2}},
$$

with $H_{1}$ concave and $H_{2}$ convex, and $\phi$ is uniformly continuous, Bardi and Faggian [1] have recently found explicit pointwise upper and lower bounds of Hopf type for the (unique) viscosity solution of (1)-(2). The upper and lower bounds are, respectively, the min-max and maxmin of a common family of explicit functions (over finite-dimensional sets of parameters), so they may be shown to coincide in some cases. In fact, using the "index of non-convexity" and a classical minimax theorem, Bardi-Faggian have thus obtained a representation formula for the viscosity solution, at least for short times (when the upper and lower bounds are equal).

In this note, we extend Bardi-Faggian's estimates to the case of general concave-convex Hamiltonians. Along the way, some remarks on their estimates simplified in a particular case where $\phi$ is Lipschitz continuous (cf. [1, Lemma 3.3]) will be given. The estimates we obtain in this general case also shed some new light on the result we have previously obtained in [7].

\section{Conjugate concave-convex functions.}

We use $|\cdot|$ and $\langle\cdot, \cdot\rangle$ to denote the Euclidean norm and scalar product, respectively, in $\mathbf{R}^{n}$. (It will cause no confusion if we use the same notation for the corresponding ones in $\mathbf{R}^{n_{1}}$, or $\mathbf{R}^{n_{2}}$, or even in any other $\mathbf{R}^{N}$.) Let $f=f(p)$ be a differentiable real-valued function on an open nonempty subset $A$ of $\mathbf{R}^{n}$. The Legendre conjugate of the pair $(A, f)$ is defined to be the pair $(B, g)$, where $B$ is the image of $A$ under the gradient mapping $\partial f / \partial p$, and $g$ is the function of $z \in B$ given by the formula

$$
g(z):=\left\langle z,(\partial f / \partial p)^{-1}(z)\right\rangle-f\left((\partial f / \partial p)^{-1}(z)\right) .
$$

It is not actually necessary to have $\partial f / \partial p$ one-to-one on $A$ in order that $g$ be well-defined (i.e., single-valued). It suffices if $\langle z, p\rangle-f(p)=\langle z, \bar{p}\rangle-f(\bar{p})$ whenever $(\partial f / \partial p)(p)=$ $(\partial f / \partial p)(\bar{p})=z$. Then the value $g(z)$ can be obtained unambiguously from the formula by replacing the set $(\partial f / \partial p)^{-1}(z)$ by any of the vectors it contains.

Passing from $(A, f)$ to the Legendre conjugate $(B, g)$, if the latter is well-defined, is called the Legendre transformation. The important role played by the Legendre transformation in the classical local theory of nonlinear equations of first-order is well-known. The global Legendre transformation has been studied extensively for convex functions. If $f$ and $A$ are convex, one can extend $f$ to be a lower semicontinuous proper convex function on all of $\mathbf{R}^{n}$ with $A$ as the interior of its effective domain. From [6, Theorem 26.4], we conclude that the Legendre conjugate $(B, g)$ of $(A, f)$ is then well-defined. Moreover, $B$ (namely the range of $\partial f / \partial p)$ is a subset of dom $f^{*}$, and $g$ is the restriction of the Fenchel conjugate $f^{*}$ to $B$. Further (cf. [6, Theorems 26.5-26.6]), when $f$ is strictly convex on $A:=\mathbf{R}^{n}$ and is co-finite, $g=f^{*}$ (i.e., $\left.B=\mathbf{R}^{n}\right)$ should be everywhere differentiable, and $(\partial f / \partial p)^{-1}(z) \equiv\{(\partial g / \partial z)(z)\}$. 
All concave-convex functions $f=f\left(p^{\prime}, p^{\prime \prime}\right)$ under our consideration are assumed to be finite and, except for the last section, to satisfy the following two "co-finiteness conditions."

$$
\begin{aligned}
& \lim _{\left|p^{\prime \prime}\right| \rightarrow+\infty} \frac{f\left(p^{\prime}, p^{\prime \prime}\right)}{\left|p^{\prime \prime}\right|}=+\infty \text { for each } p^{\prime} \in \mathbf{R}^{n_{1}}, \\
& \lim _{\left|p^{\prime}\right| \rightarrow+\infty} \frac{f\left(p^{\prime}, p^{\prime \prime}\right)}{\left|p^{\prime}\right|}=-\infty \text { for each } p^{\prime \prime} \in \mathbf{R}^{n_{2}} .
\end{aligned}
$$

Let $f^{*_{2}}=f^{*_{2}}\left(p^{\prime}, z^{\prime \prime}\right)$ (resp. $\left.f^{*_{1}}=f^{*_{1}}\left(z^{\prime}, p^{\prime \prime}\right)\right)$ be, for each fixed $p^{\prime} \in \mathbf{R}^{n_{1}}$ (resp. $p^{\prime \prime} \in$ $\mathbf{R}^{n_{2}}$ ), the Fenchel conjugate of a given $p^{\prime \prime}$-convex (resp. $p^{\prime}$-concave) function $f=f\left(p^{\prime}, p^{\prime \prime}\right)$. In other words,

$$
\begin{aligned}
f^{*_{2}}\left(p^{\prime}, z^{\prime \prime}\right) & :=\sup _{p^{\prime \prime} \in \mathbf{R}^{n_{2}}}\left\{\left\langle z^{\prime \prime}, p^{\prime \prime}\right\rangle-f\left(p^{\prime}, p^{\prime \prime}\right)\right\} \\
\text { (resp. } f^{*_{1}}\left(z^{\prime}, p^{\prime \prime}\right) & \left.:=\inf _{p^{\prime} \in \mathbf{R}^{n_{1}}}\left\{\left\langle z^{\prime}, p^{\prime}\right\rangle-f\left(p^{\prime}, p^{\prime \prime}\right)\right\}\right)
\end{aligned}
$$

for $\left(p^{\prime}, z^{\prime \prime}\right) \in \mathbf{R}^{n_{1}} \times \mathbf{R}^{n_{2}}$ (resp. $\left(z^{\prime}, p^{\prime \prime}\right) \in \mathbf{R}^{n_{1}} \times \mathbf{R}^{n_{2}}$ ). If $f$ is concave-convex, then (5) (resp. (6)) actually implies the convexity (resp. concavity) of $f^{*_{2}}$ (resp. $f^{*_{1}}$ ) not only in $z^{\prime \prime} \in \mathbf{R}^{n_{2}}$ (resp. $z^{\prime} \in \mathbf{R}^{n_{1}}$ ) but also in the whole variable $\left(p^{\prime}, z^{\prime \prime}\right) \in \mathbf{R}^{n_{1}} \times \mathbf{R}^{n_{2}}$ (resp. $\left(z^{\prime}, p^{\prime \prime}\right) \in$ $\mathbf{R}^{n_{1}} \times \mathbf{R}^{n_{2}}$ ). Moreover, under the condition (3) (resp. (4)), the finiteness of $f$ clearly yields that of $f^{*_{2}}$ (resp. $f^{*_{1}}$ ) with

$$
\lim _{\left|z^{\prime \prime}\right| \rightarrow+\infty} \frac{f^{*_{2}}\left(p^{\prime}, z^{\prime \prime}\right)}{\left|z^{\prime \prime}\right|}=+\infty \quad\left(\text { resp. } \lim _{\left|z^{\prime}\right| \rightarrow+\infty} \frac{f^{*_{1}}\left(z^{\prime}, p^{\prime \prime}\right)}{\left|z^{\prime}\right|}=-\infty\right)
$$

locally uniformly in $p^{\prime} \in \mathbf{R}^{n_{1}}$ (resp. $p^{\prime \prime} \in \mathbf{R}^{n_{2}}$ ).

If (4) (resp. (3)) is satisfied, then (5) (resp. (6)) gives

$$
\begin{gathered}
\frac{f^{*_{2}}\left(p^{\prime}, z^{\prime \prime}\right)}{\left|p^{\prime}\right|} \geq-\frac{f\left(p^{\prime}, 0^{\prime \prime}\right)}{\left|p^{\prime}\right|} \rightarrow+\infty \quad \text { as }\left|p^{\prime}\right| \rightarrow+\infty \\
\left(\operatorname{resp} . \frac{f^{*_{1}}\left(z^{\prime}, p^{\prime \prime}\right)}{\left|p^{\prime \prime}\right|} \leq-\frac{f\left(0^{\prime}, p^{\prime \prime}\right)}{\left|p^{\prime \prime}\right|} \rightarrow-\infty \quad \text { as }\left|p^{\prime \prime}\right| \rightarrow+\infty\right)
\end{gathered}
$$

uniformly in $z^{\prime \prime} \in \mathbf{R}^{n_{2}}$ (resp. $z^{\prime} \in \mathbf{R}^{n_{1}}$ ).

Now let $f$ be concave-convex. Besides the Fenchel "partial conjugates" $f^{*_{2}}$ and $f^{*_{1}}$, we shall consider the following two "total conjugates" of $f$. The first one, which we denote by $\bar{f}^{*}=\bar{f}^{*}\left(z^{\prime}, z^{\prime \prime}\right)$, is defined as the Fenchel conjugate of the concave function $\mathbf{R}^{n_{1}} \ni p^{\prime} \mapsto$ $-f^{*_{2}}\left(p^{\prime}, z^{\prime \prime}\right)$ for each fixed $z^{\prime \prime} \in \mathbf{R}^{n_{2}}$; more precisely,

$$
\bar{f}^{*}\left(z^{\prime}, z^{\prime \prime}\right):=\inf _{p^{\prime} \in \mathbf{R}^{n_{1}}}\left\{\left\langle z^{\prime}, p^{\prime}\right\rangle+f^{*_{2}}\left(p^{\prime}, z^{\prime \prime}\right)\right\}
$$

The second, $\underline{f}^{*}=\underline{f}^{*}\left(z^{\prime}, z^{\prime \prime}\right)$, is defined as the Fenchel conjugate of the convex function $\mathbf{R}^{n_{2}} \ni p^{\prime \prime} \mapsto-f^{*_{1}}\left(\overline{z^{\prime}}, p^{\prime \prime}\right)$ for each fixed $z^{\prime} \in \mathbf{R}^{n_{1}}$; i.e.,

$$
\underline{f}^{*}\left(z^{\prime}, z^{\prime \prime}\right):=\sup _{p^{\prime \prime} \in \mathbf{R}^{n_{2}}}\left\{\left\langle z^{\prime \prime}, p^{\prime \prime}\right\rangle+f^{* 1}\left(z^{\prime}, p^{\prime \prime}\right)\right\} .
$$


By (5)-(6) and (9)-(10), we have

$$
\begin{aligned}
& \bar{f}^{*}\left(z^{\prime}, z^{\prime \prime}\right)=\inf _{p^{\prime} \in \mathbf{R}^{n_{1}}} \sup _{p^{\prime \prime} \in \mathbf{R}^{n_{2}}}\left\{\left\langle z^{\prime}, p^{\prime}\right\rangle+\left\langle z^{\prime \prime}, p^{\prime \prime}\right\rangle-f\left(p^{\prime}, p^{\prime \prime}\right)\right\}, \\
& \underline{f}^{*}\left(z^{\prime}, z^{\prime \prime}\right)=\sup _{p^{\prime \prime} \in \mathbf{R}^{n_{2}}} \inf _{p^{\prime} \in \mathbf{R}^{n_{1}}}\left\{\left\langle z^{\prime}, p^{\prime}\right\rangle+\left\langle z^{\prime \prime}, p^{\prime \prime}\right\rangle-f\left(p^{\prime}, p^{\prime \prime}\right)\right\} .
\end{aligned}
$$

Therefore, the functions $\bar{f}^{*}$ and $\underline{f}^{*}$ will usually be called the upper and lower conjugates, respectively, of $f$. (Of course, (11)-(12) imply $\bar{f}^{*} \geq \underline{f}^{*}$.) For any $z^{\prime} \in \mathbf{R}^{n_{1}}$,

$$
\mathbf{R}^{n_{1}} \times \mathbf{R}^{n_{2}} \ni\left(p^{\prime}, z^{\prime \prime}\right) \mapsto h\left(p^{\prime}, z^{\prime \prime}\right):=\left\langle z^{\prime}, p^{\prime}\right\rangle+f^{*_{2}}\left(p^{\prime}, z^{\prime \prime}\right)
$$

is a convex function. But (9) shows that $\bar{f}^{*}\left(z^{\prime}, \cdot\right)$ is the image $A h$,

$$
(A h)\left(z^{\prime \prime}\right):=\inf \left\{h\left(p^{\prime}, z^{\prime \prime}\right): A\left(p^{\prime}, z^{\prime \prime}\right)=z^{\prime \prime}\right\},
$$

of $h$ under the (linear) projection $\mathbf{R}^{n_{1}} \times \mathbf{R}^{n_{2}} \ni\left(p^{\prime}, z^{\prime \prime}\right) \mapsto A\left(p^{\prime}, z^{\prime \prime}\right):=z^{\prime \prime}$. It follows from [6, Theorem 5.7] that $\bar{f}^{*}\left(z^{\prime}, z^{\prime \prime}\right)$ is convex in $z^{\prime \prime} \in \mathbf{R}^{n_{2}}$. On the other hand, by definition, $\bar{f}^{*}\left(z^{\prime}, z^{\prime \prime}\right)$ is necessarily concave in $z^{\prime} \in \mathbf{R}^{n_{1}}$. The upper conjugate $\bar{f}^{*}$ is hence a concaveconvex function on $\mathbf{R}^{n_{1}} \times \mathbf{R}^{n_{2}}$. The same conclusion may dually be drawn for the lower conjugate $\underline{f}^{*}$.

We have previously seen that if the concave-convex function $f$ is finite on $\mathbf{R}^{n_{1}} \times \mathbf{R}^{n_{2}}$ and satisfies (3)-(4), its partial conjugates $f^{*_{2}}$ and $f^{*_{1}}$ must both be finite with (7)-(8) holding. Thus, by (9)-(10), the "total conjugates" $\bar{f}^{*}$ and $\underline{f}^{*}$ are then also finite; and hence they coincide [6, Corollary 37.1.2]. In this situation, the Fenchel conjugate

$$
f^{*}:=\bar{f}^{*}=\underline{f}^{*}
$$

of $f$ will simultaneously has the properties:

$$
\begin{aligned}
& \lim _{\left|z^{\prime \prime}\right| \rightarrow+\infty} \frac{f^{*}\left(z^{\prime}, z^{\prime \prime}\right)}{\left|z^{\prime \prime}\right|}=+\infty \quad \text { for each } z^{\prime} \in \mathbf{R}^{n_{1}}, \\
& \lim _{\left|z^{\prime}\right| \rightarrow+\infty} \frac{f^{*}\left(z^{\prime}, z^{\prime \prime}\right)}{\left|z^{\prime}\right|}=-\infty \quad \text { for each } z^{\prime \prime} \in \mathbf{R}^{n_{2}} .
\end{aligned}
$$

(If (3)-(4) are not assumed, $\bar{f}^{*}$ and $\underline{f}^{*}$ may not coincide. Then, in accordance with the terminology of Rockafellar, any concave-convex function $g$ that lies between $\bar{f}^{*}$ and $\underline{f}^{*}$ is called a conjugate of our Hamiltonian $f$.)

A finite concave-convex function $f=f\left(p^{\prime}, p^{\prime \prime}\right)$ on $\mathbf{R}^{n_{1}} \times \mathbf{R}^{n_{2}}$ is said to be strict if its concavity in $p^{\prime} \in \mathbf{R}^{n_{1}}$ and convexity in $p^{\prime \prime} \in \mathbf{R}^{n_{2}}$ are both strict. It will then also be called a strictly concave-convex function on $\mathbf{R}^{n_{1}} \times \mathbf{R}^{n_{2}}$. The global Legendre transformation in a class of strictly concave-convex functions can be described via their Fenchel conjugates as follows. (For the proofs, see [7, Chapter 10].)

LEMMA 1. Let $f=f\left(p^{\prime}, p^{\prime \prime}\right)$ be a strictly concave-convex function on $\mathbf{R}^{n_{1}} \times \mathbf{R}^{n_{2}}$ with (3) (resp. (4)) holding. Then its partial conjugate $f^{*_{2}}=f^{*_{2}}\left(p^{\prime}, z^{\prime \prime}\right)$ (resp. $f^{*_{1}}=$ $f^{*_{1}}\left(z^{\prime}, p^{\prime \prime}\right)$ ) defined by (5) (resp. (6)) is strictly convex (resp. concave) in $p^{\prime} \in \mathbf{R}^{n_{1}}$ (resp. 
$p^{\prime \prime} \in \mathbf{R}^{n_{2}}$ ) and everywhere differentiable in $z^{\prime \prime} \in \mathbf{R}^{n_{2}}$ (resp. $z^{\prime} \in \mathbf{R}^{n_{1}}$ ). Besides that, $\partial f^{*_{2}} / \partial z^{\prime \prime}$ (resp. $\partial f^{*_{1}} / \partial z^{\prime}$ ) is continuous on $\mathbf{R}^{n_{1}} \times \mathbf{R}^{n_{2}}$ with

$$
\begin{aligned}
f^{*_{2}}\left(p^{\prime}, z^{\prime \prime}\right) & \equiv\left\langle z^{\prime \prime},\left(\partial f^{*_{2}} / \partial z^{\prime \prime}\right)\left(p^{\prime}, z^{\prime \prime}\right)\right\rangle-f\left(p^{\prime},\left(\partial f^{*_{2}} / \partial z^{\prime \prime}\right)\left(p^{\prime}, z^{\prime \prime}\right)\right) \\
\text { (resp. } f^{*_{1}}\left(z^{\prime}, p^{\prime \prime}\right) & \left.\equiv\left\langle z^{\prime},\left(\partial f^{*_{1}} / \partial z^{\prime}\right)\left(z^{\prime}, p^{\prime \prime}\right)\right\rangle-f\left(\left(\partial f^{*_{1}} / \partial z^{\prime}\right)\left(z^{\prime}, p^{\prime \prime}\right), p^{\prime \prime}\right)\right) .
\end{aligned}
$$

PROPOSITION 1. Let $f=f\left(p^{\prime}, p^{\prime \prime}\right)$ be a strictly concave-convex function on $\mathbf{R}^{n_{1}} \times$ $\mathbf{R}^{n_{2}}$ with both (3) and (4) holding. Then its conjugate $f^{*}=f^{*}\left(z^{\prime}, z^{\prime \prime}\right)$ defined by (9)(13) is likewise a concave-convex function satisfying $\left(3^{*}\right)-\left(4^{*}\right)$. Moreover, $f^{*}$ is everywhere continuously differentiable with

$$
f^{*}(z) \equiv\left\langle z,\left(\partial f^{*} / \partial z\right)(z)\right\rangle-f\left(\left(\partial f^{*} / \partial z\right)(z)\right) .
$$

\section{A Hopf-type formula.}

We now consider the Cauchy problem

$$
\begin{aligned}
\partial u / \partial t+f(\partial u / \partial x) & =0 \quad \text { in } \mathcal{D}:=\left\{t>0, x=\left(x^{\prime}, x^{\prime \prime}\right) \in \mathbf{R}^{n_{1}} \times \mathbf{R}^{n_{2}}\right\}, \\
u(0, x) & =\phi(x) \text { on }\left\{t=0, x \in \mathbf{R}^{n_{1}} \times \mathbf{R}^{n_{2}}\right\} .
\end{aligned}
$$

An explicit global solution of the problem can be found under the next three hypotheses.

(I) The initial function $\phi$ is continuous and the Hamiltonian $f=f\left(p^{\prime}, p^{\prime \prime}\right)$ is strictly concave-convex on $\mathbf{R}^{n_{1}} \times \mathbf{R}^{n_{2}}$ with (3)-(4) holding.

(II) The equality $\sup _{y^{\prime} \in \mathbf{R}^{n_{1}}} \inf _{y^{\prime \prime} \in \mathbf{R}^{n_{2}}} \zeta(t, x, y)=\inf _{y^{\prime \prime} \in \mathbf{R}^{n_{2}}} \sup _{y^{\prime} \in \mathbf{R}^{n_{1}}} \zeta(t, x, y)$ is satisfied in $\mathcal{D}$, where

$$
\zeta(t, x, y):=\phi(y)+t \cdot f^{*}((x-y) / t)
$$

for $(t, x) \in \mathcal{D}, y=\left(y^{\prime}, y^{\prime \prime}\right) \in \mathbf{R}^{n_{1}} \times \mathbf{R}^{n_{2}}$. Here, $f^{*}$ denotes the conjugate defined by (9)-(13) off. that

(III) To each bounded subset $V$ of $\mathcal{D}$ there corresponds a positive number $M(V)$ so

$$
\begin{gathered}
\min _{\substack{w^{\prime \prime} \in \mathbf{R}^{n_{2}} \\
\left|w^{\prime \prime}\right| \leq M(V)}} \sup _{w^{\prime} \in \mathbf{R}^{n_{1}}} \zeta\left(t, x, w^{\prime}, w^{\prime \prime}\right)<\sup _{w^{\prime} \in \mathbf{R}^{n_{1}}} \zeta\left(t, x, w^{\prime}, y^{\prime \prime}\right), \\
\max _{\substack{w^{\prime} \in \mathbf{R}^{n_{1}} \\
\left|w^{\prime}\right| \leq M(V)}} \inf _{w^{\prime \prime} \in \mathbf{R}^{n_{2}}} \zeta\left(t, x, w^{\prime}, w^{\prime \prime}\right)>\inf _{w^{\prime \prime} \in \mathbf{R}^{n_{2}}} \zeta\left(t, x, y^{\prime}, w^{\prime \prime}\right)
\end{gathered}
$$

whenever $(t, x) \in V$ and $\min \left\{\left|y^{\prime}\right|,\left|y^{\prime \prime}\right|\right\}>M(V)$.

Actually, we have proved in [7] the following:

THEOREM 1 [7, Theorem 10.4]. Assume (I)-(III). Then the formula

$$
u(t, x):=\sup _{y^{\prime} \in \mathbf{R}^{n_{1}}} \inf _{y^{\prime \prime} \in \mathbf{R}^{n_{2}}} \zeta(t, x, y)=\inf _{y^{\prime \prime} \in \mathbf{R}^{n_{2}}} \sup _{y^{\prime} \in \mathbf{R}^{n_{1}}} \zeta(t, x, y)
$$

( for $(t, x) \in \mathcal{D}$ ) determines a global solution of the Cauchy problem (14)-(15).

REMARK 1. Our hypotheses imply that a "saddle-point" of the function $\zeta(t, x, \cdot)$ given by (16) (with respect to maximizing over $\mathbf{R}^{n_{1}}$ and minimizing over $\mathbf{R}^{n_{2}}$ ) exists. If $f$ has a 
special representation $f\left(p^{\prime}, p^{\prime \prime}\right):=H_{1}\left(p^{\prime}\right)+H_{2}\left(p^{\prime \prime}\right)$ on $\mathbf{R}^{n_{1}} \times \mathbf{R}^{n_{2}}$, with $H_{1}$ concave and $\mathrm{H}_{2}$ convex, then one can use the "index of non-convexity" and a classical minimax theorem to give sufficient conditions for (II) to hold. (Concerning this question, see Bardi and Faggian [1].)

COROLLARY 1. Under Hypotheses (I)-(II), suppose that

$$
\begin{aligned}
& \liminf _{\left|x^{\prime \prime}\right| \rightarrow+\infty} \frac{\phi\left(x^{\prime}, x^{\prime \prime}\right)}{\left|x^{\prime \prime}\right|}>-\infty \quad \text { locally uniformly in } x^{\prime} \in \mathbf{R}^{n_{1}}, \\
& \limsup _{\left|x^{\prime}\right| \rightarrow+\infty} \frac{\phi\left(x^{\prime}, x^{\prime \prime}\right)}{\left|x^{\prime}\right|}<+\infty \quad \text { locally uniformly in } x^{\prime \prime} \in \mathbf{R}^{n_{2}} .
\end{aligned}
$$

Then (17) determines a global solution of the Cauchy problem (14)-(15).

REMARK 2. If $n_{1}=0$ or $n_{2}=0$, the classical Hopf formula for convex or concave Hamiltonians will be obtained from (17). In this case, (II) holds trivially. Thus, one can see from Corollary 1 that our assumption on the initial data is weaker than that of Hopf: for the inequalities in this corollary to hold, $\phi$ needs not be (globally) Lipschitz continuous (but it is required so by Hopf [3] for the validity of his formula). We may easily check this, using the following concrete examples: (i) $\phi(x):=\prod_{i=1}^{n} \sin x_{i}^{2}$; and (ii) $\phi(x):= \pm \sqrt{|x|}$.

\section{Hopf-type estimates for viscosity solutions.}

We still consider the Cauchy problem (14)-(15), but throughout this section $\phi$ is uniformly continuous, and $f=f\left(p^{\prime}, p^{\prime \prime}\right)$ is a general finite concave-convex function. Then this Hamiltonian $f$ is continuous by [6, Theorem 35.1]. Therefore, it is known (see [2], [4]) that the problem under consideration has a unique viscosity solution $u=u(t, x)$ in the space $U C_{x}\left([0,+\infty) \times \mathbf{R}^{n}\right)$ of the continuous functions which are uniformly continuous in $x$ uniformly in $t$.

Without (3) (resp. (4)), the partial conjugate $f^{*_{2}}$ (resp. $f^{*_{1}}$ ) defined in (5) (resp. (6)) is still, of course, convex (resp. concave), but might be infinite somewhere. One can claim only that

$$
\begin{aligned}
f^{*_{2}}\left(p^{\prime}, z^{\prime \prime}\right)>-\infty & \forall\left(p^{\prime}, z^{\prime \prime}\right) \in \mathbf{R}^{n_{1}} \times \mathbf{R}^{n_{2}} \\
\text { (resp. } f^{*_{1}}\left(z^{\prime}, p^{\prime \prime}\right)<+\infty & \left.\forall\left(z^{\prime}, p^{\prime \prime}\right) \in \mathbf{R}^{n_{1}} \times \mathbf{R}^{n_{2}}\right)
\end{aligned}
$$

Now, let

$$
\begin{aligned}
D_{1}: & =\left\{z^{\prime} \in \mathbf{R}^{n_{1}}: f^{*_{1}}\left(z^{\prime}, p^{\prime \prime}\right)>-\infty \quad \forall p^{\prime \prime} \in \mathbf{R}^{n_{2}}\right\} \\
& =\left\{z^{\prime} \in \mathbf{R}^{n_{1}}: \operatorname{dom} f^{*_{1}}\left(z^{\prime}, \cdot\right)=\mathbf{R}^{n_{2}}\right\}=\bigcap_{p^{\prime \prime} \in \mathbf{R}^{n_{2}}} \operatorname{dom} f^{*_{1}}\left(\cdot, p^{\prime \prime}\right), \\
D_{2}: & =\left\{z^{\prime \prime} \in \mathbf{R}^{n_{2}}: f^{*_{2}}\left(p^{\prime}, z^{\prime \prime}\right)<+\infty \quad \forall p^{\prime} \in \mathbf{R}^{n_{1}}\right\} \\
& =\left\{z^{\prime \prime} \in \mathbf{R}^{n_{2}}: \operatorname{dom} f^{*_{2}}\left(\cdot, z^{\prime \prime}\right)=\mathbf{R}^{n_{1}}\right\}=\bigcap_{p^{\prime} \in \mathbf{R}^{n_{1}}} \operatorname{dom} f^{*_{2}}\left(p^{\prime}, \cdot\right),
\end{aligned}
$$


and, for $(t, x) \in \overline{\mathcal{D}}$, set

$$
\begin{aligned}
& u_{-}(t, x):=\sup _{z^{\prime} \in D_{1}} \min _{z^{\prime \prime} \in \mathbf{R}^{n_{2}}}\left\{\phi(x-t z)+t \cdot \underline{f}^{*}(z)\right\}, \\
& u_{+}(t, x):=\inf _{z^{\prime \prime} \in D_{2}} \max _{z^{\prime} \in \mathbf{R}^{n_{1}}}\left\{\phi(x-t z)+t \cdot \bar{f}^{*}(z)\right\},
\end{aligned}
$$

where the lower and upper conjugates, $f^{*}$ and $\bar{f}^{*}$, of the Hamiltonian $f$ are the concaveconvex functions (with possibly infinite values) defined by (9)-(12). Clearly, if $(t, x) \in \mathcal{D}$, we also have

$$
\begin{aligned}
& u_{-}(t, x)=\sup _{y^{\prime} \in x^{\prime}-t \cdot D_{1}} \min _{y^{\prime \prime} \in \mathbf{R}^{n_{2}}}\left\{\phi(y)+t \cdot \underline{f}^{*}((x-y) / t)\right\}, \\
& u_{+}(t, x)=\inf _{y^{\prime \prime} \in x^{\prime \prime}-t \cdot D_{2}} \max _{y^{\prime} \in \mathbf{R}^{n_{1}}}\left\{\phi(y)+t \cdot \bar{f}^{*}((x-y) / t)\right\},
\end{aligned}
$$

cf. (16)-(17). Our estimates for viscosity solutions in the case of general concave-convex Hamiltonians read as follows:

THEOREM 2. Let $f$ be a ( finite) concave-convex function, and $\phi$ be uniformly continuous. Then the unique viscosity solution $u \in U C_{x}\left([0,+\infty) \times \mathbf{R}^{n}\right)$ of the Cauchy problem (14)-(15) satisfies on $\overline{\mathcal{D}}$ the inequalities

$$
u_{-}(t, x) \leq u(t, x) \leq u_{+}(t, x),
$$

where $u_{-}$and $u_{+}$are defined by (20)-(21).

Proof. For each $\underline{z}^{\prime} \in D_{1}$, let $F\left(p, \underline{z}^{\prime}\right)=F\left(p^{\prime}, p^{\prime \prime}, \underline{z}^{\prime}\right):=\left\langle\underline{z}^{\prime}, p^{\prime}\right\rangle-f^{*_{1}}\left(\underline{z}^{\prime}, p^{\prime \prime}\right)$. Then $F\left(\cdot, \underline{z}^{\prime}\right)$ is a (finite) convex function on $\mathbf{R}^{n}$ with its (Fenchel) conjugate $F^{*}\left(\cdot, \underline{z}^{\prime}\right)$ given (cf. (10)) by

$$
\begin{aligned}
F^{*}\left(z, \underline{z}^{\prime}\right) & =\sup _{p \in \mathbf{R}^{n}}\left\{\langle z, p\rangle-\left\langle\underline{z}^{\prime}, p^{\prime}\right\rangle+f^{* 1}\left(\underline{z}^{\prime}, p^{\prime \prime}\right)\right\} \\
& = \begin{cases}+\infty & \text { if } z=\left(z^{\prime}, z^{\prime \prime}\right) \neq\left(\underline{z}^{\prime}, z^{\prime \prime}\right), \\
\underline{f}^{*}\left(\underline{z}^{\prime}, z^{\prime \prime}\right) & \text { if } z=\left(\underline{z}^{\prime}, z^{\prime \prime}\right) .\end{cases}
\end{aligned}
$$

Next, consider the Cauchy problem

$$
\begin{gathered}
\partial \psi / \partial t+F\left(\partial \psi / \partial x, \underline{z}^{\prime}\right)=0 \quad \text { in }\left\{t>0, x \in \mathbf{R}^{n}\right\}, \\
\psi\left(0, x, \underline{z}^{\prime}\right)=\phi(x) \text { on }\left\{t=0, x \in \mathbf{R}^{n}\right\} .
\end{gathered}
$$

This is the Cauchy problem for a convex Hamilton-Jacobi equation (with uniformly continuous initial data). In view of (22), its (unique) viscosity solution $\psi\left(\cdot, \underline{z}^{\prime}\right)$ can be represented [1, Theorem 2.1] as

$$
\begin{aligned}
\psi\left(t, x, \underline{z}^{\prime}\right) & =\min _{z \in \mathbf{R}^{n}}\left\{\phi(x-t z)+t \cdot F^{*}\left(z, \underline{z}^{\prime}\right)\right\} \\
& =\min _{z^{\prime \prime} \in \mathbf{R}^{n_{2}}}\left\{\phi\left(x^{\prime}-t \underline{z}^{\prime}, x^{\prime \prime}-t z^{\prime \prime}\right)+t \cdot \underline{f}^{*}\left(\underline{z}^{\prime}, z^{\prime \prime}\right)\right\}
\end{aligned}
$$


Since $f\left(p^{\prime}, p^{\prime \prime}\right) \leq\left\langle\underline{z}^{\prime}, p^{\prime}\right\rangle-f^{*_{1}}\left(\underline{z}^{\prime}, p^{\prime \prime}\right)=F\left(p^{\prime}, p^{\prime \prime}, \underline{z}^{\prime}\right)$, we may prove that $\psi\left(\cdot, \underline{z}^{\prime}\right)$ is a viscosity subsolution of (14)-(15). (In fact, let $\varphi \in C^{1}(\mathcal{D})$ be a test function such that $\psi\left(\cdot, \underline{z}^{\prime}\right)-\varphi$ has a local maximum at some $(t, x) \in \mathcal{D}$. Then

$$
\partial \varphi / \partial t+f(\partial \varphi / \partial x) \leq \partial \varphi / \partial t+F\left(\partial \varphi / \partial x, \underline{z}^{\prime}\right) \leq 0 \text { at }(t, x),
$$

as claimed.)

Now, a standard comparison theorem for unbounded viscosity solutions (see [2]) gives

$$
\psi\left(t, x, z^{\prime}\right) \leq u(t, x) \quad \forall z^{\prime} \in D_{1} .
$$

Hence, by (20) and (23), $u_{-}(t, x)=\sup _{z^{\prime} \in D_{1}} \psi\left(t, x, z^{\prime}\right) \leq u(t, x)$ for all $(t, x) \in \overline{\mathcal{D}}$. Dually, we have $u_{+}(t, x) \geq u(t, x)$ on $\overline{\mathcal{D}}$.

REMARK 3. It can be shown that $u_{-}$(resp. $u_{+}$) is a subsolution (resp. supersolution) of (14)-(15) in the generalized sense of Ishii [5], provided $D_{1} \neq \emptyset$ (resp. $\left.D_{2} \neq \emptyset\right)$, cf. (18)(19). Further, let $f\left(p^{\prime}, p^{\prime \prime}\right) \equiv H_{1}\left(p^{\prime}\right)+H_{2}\left(p^{\prime \prime}\right)$, with $H_{1}$ concave, $H_{2}$ convex (both finite). As a consequence of Theorem 2 , we then see that the (unique) viscosity solution $u$ of the Cauchy problem (14)-(15) satisfies on $\overline{\mathcal{D}}$ the inequalities

$$
\begin{gathered}
\max _{z^{\prime} \in \mathbf{R}^{n_{1}}} \min _{z^{\prime \prime} \in \mathbf{R}^{n_{2}}}\left\{\phi(x-t z)+t \cdot\left(H_{1}^{*}\left(z^{\prime}\right)+H_{2}^{*}\left(z^{\prime \prime}\right)\right)\right\} \leq u(t, x) \\
\leq \min _{z^{\prime \prime} \in \mathbf{R}^{n_{2}}} \max _{z^{\prime} \in \mathbf{R}^{n_{1}}}\left\{\phi(x-t z)+t \cdot\left(H_{1}^{*}\left(z^{\prime}\right)+H_{2}^{*}\left(z^{\prime \prime}\right)\right)\right\} .
\end{gathered}
$$

These are essentially Bardi-Faggian's estimates [1, (3.7)] (with only differences in notation). Here, we follow Rockafellar $[6, \S 30]$ to take

$$
H_{1}^{*}\left(z^{\prime}\right):=\inf _{p^{\prime} \in \mathbf{R}^{n_{1}}}\left\{\left\langle z^{\prime}, p^{\prime}\right\rangle-H_{1}\left(p^{\prime}\right)\right\}, \quad \text { while } H_{2}^{*}\left(z^{\prime \prime}\right):=\sup _{p^{\prime \prime} \in \mathbf{R}^{n_{2}}}\left\{\left\langle z^{\prime \prime}, p^{\prime \prime}\right\rangle-H_{2}\left(p^{\prime \prime}\right)\right\} .
$$

(Caution: in general, $H_{1}^{*} \neq-\left(-H_{1}\right)^{*}$. For the convex function $G:=-H_{1}$, one has, not $H_{1}^{*}\left(z^{\prime}\right)=-G^{*}\left(z^{\prime}\right)$, but $H_{1}^{*}\left(z^{\prime}\right)=-G^{*}\left(-z^{\prime}\right)$.)

Of course, for $t \cdot\left(H_{1}^{*}\left(z^{\prime}\right)+H_{2}^{*}\left(z^{\prime \prime}\right)\right)$ not to be vague (and the desired estimates to hold), we adopt the convention that $0 \cdot( \pm \infty)=0$, and we may set $H_{1}^{*}\left(z^{\prime}\right)+H_{2}^{*}\left(z^{\prime \prime}\right)=-\infty+\infty$ to be any value in $[-\infty,+\infty]$ if $z^{\prime} \notin \operatorname{dom} H_{1}^{*}, z^{\prime \prime} \notin \operatorname{dom} H_{2}^{*}$. However, "max" and "min" in the above Bardi-Faggian estimates are actually attained on $D_{1} \equiv \operatorname{dom} H_{1}^{*}:=\left\{z^{\prime} \in \mathbf{R}^{n_{1}}\right.$ : $\left.H_{1}^{*}\left(z^{\prime}\right)>-\infty\right\}$ and $D_{2} \equiv \operatorname{dom} H_{2}^{*}:=\left\{z^{\prime \prime} \in \mathbf{R}^{n_{2}}: H_{2}^{*}\left(z^{\prime \prime}\right)<+\infty\right\}$, respectively.

Going back to Theorem 1, we now have:

COROllary 2. Assume (I)-(II) (see §3), with $\phi$ uniformly continuous. Then (17) determines the (unique) viscosity solution of the Cauchy problem (14)-(15).

REMARK 4. Since $\phi$ is uniformly continuous, the inequalities in Corollary 1 are satisfied. This implies that the viscosity solution is locally Lipschitz continuous and solves (14) almost everywhere. Notice also that here we have $D_{1} \equiv \mathbf{R}^{n_{1}}$ and $D_{2} \equiv \mathbf{R}^{n_{2}}$ (cf. (18)-(19)). 
If $\phi$ is Lipschitz continuous, then "min" and "max" in (20) and (21) can be computed on particular compact subsets of $\mathbf{R}^{n_{2}}$ and $\mathbf{R}^{n_{1}}$, respectively. In fact, we have the following, where $\operatorname{Lip}(\phi)$ stands for the Lipschitz constant of $\phi$.

LEMMA 2. Let $\phi$ be (globally) Lipschitz continuous, $f$ be (finite and) concave-convex, $L \geq 0$ be such that, for some $r>\operatorname{Lip}(\phi)$,

$$
\begin{aligned}
\left|f\left(p^{\prime}, p^{\prime \prime}\right)-f\left(p^{\prime}, \bar{p}^{\prime \prime}\right)\right| \leq L\left|p^{\prime \prime}-\bar{p}^{\prime \prime}\right| \quad \forall p^{\prime} \in \mathbf{R}^{n_{1}} ; p^{\prime \prime}, \bar{p}^{\prime \prime} \in \mathbf{R}^{n_{2}},\left|p^{\prime \prime}\right|,\left|\bar{p}^{\prime \prime}\right| \leq r \\
\text { (resp. } \left.\left|f\left(p^{\prime}, p^{\prime \prime}\right)-f\left(\bar{p}^{\prime}, p^{\prime \prime}\right)\right| \leq L\left|p^{\prime}-\bar{p}^{\prime}\right| \quad \forall p^{\prime \prime} \in \mathbf{R}^{n_{2}} ; p^{\prime}, \bar{p}^{\prime} \in \mathbf{R}^{n_{1}},\left|p^{\prime}\right|,\left|\bar{p}^{\prime}\right| \leq r\right) .
\end{aligned}
$$

Then (20) (resp. (21)) becomes

$$
\begin{aligned}
u_{-}(t, x) & =\sup _{z^{\prime} \in D_{1}} \min _{\left|z^{\prime \prime}\right| \leq L}\left\{\phi(x-t z)+t \cdot \underline{f}^{*}(z)\right\} \\
\text { (resp. } u_{+}(t, x) & \left.=\inf _{z^{\prime \prime} \in D_{2}} \max _{\left|z^{\prime}\right| \leq L}\left\{\phi(x-t z)+t \cdot \bar{f}^{*}(z)\right\}\right) .
\end{aligned}
$$

To prove Lemma 2, we need the following preparations. Given any convex Hamiltonian $H=H(q)$, and any uniformly continuous initial data $v_{0}=v_{0}(\alpha)\left(\alpha, q \in \mathbf{R}^{N}\right)$, as was already mentioned, the Hopf formula

$$
v(t, \alpha):=\min _{\omega \in \mathbf{R}^{N}}\left\{v_{0}(\alpha-t \omega)+t \cdot H^{*}(\omega)\right\} \quad\left(t \geq 0, \alpha \in \mathbf{R}^{N}\right)
$$

determines the unique viscosity solution $v=v(t, \alpha)$ in the space $U C_{\alpha}\left([0,+\infty) \times \mathbf{R}^{N}\right)$ of the Cauchy problem

$$
\begin{gathered}
\partial v / \partial t+H(\partial v / \partial \alpha)=0 \quad \text { in }\left\{t>0, \alpha \in \mathbf{R}^{N}\right\}, \\
v(0, \alpha)=v_{0}(\alpha) \text { on }\left\{t=0, \alpha \in \mathbf{R}^{N}\right\}
\end{gathered}
$$

The next technical lemma is somehow related to the so-called "cone of dependence" for viscosity solutions.

LEMMA 3. Let $H$ be convex, $v_{0}$ be (globally) Lipschitz continuous. Assume that

$$
|H(q)-H(\bar{q})| \leq L|q-\bar{q}| \quad \forall q, \bar{q} \in \mathbf{R}^{N}, \quad|q|,|\bar{q}| \leq r
$$

for some $L \geq 0, r>\operatorname{Lip}\left(v_{0}\right)$. Then (24) becomes

$$
v(t, \alpha)=\min _{|\omega| \leq L}\left\{v_{0}(\alpha-t \omega)+t \cdot H^{*}(\omega)\right\} \quad\left(t \geq 0, \alpha \in \mathbf{R}^{N}\right)
$$

Proof of Lemma 3. We may suppose $t>0$. Choose $\omega_{0}=\omega_{0}(t, \alpha) \in \mathbf{R}^{N}$ so that the value at $(t, \alpha)$ of the viscosity solution $v$, determined by (24), is

$$
v_{0}\left(\alpha-t \omega_{0}\right)+t \cdot H^{*}\left(\omega_{0}\right) .
$$

It suffices to prove $\left|\omega_{0}\right| \leq L$. To this end, we first notice that

$$
v_{0}\left(\alpha-t \omega_{0}\right)+t \cdot H^{*}\left(\omega_{0}\right) \leq v_{0}(\alpha-t \omega)+t \cdot H^{*}(\omega)
$$


for any $\omega \in \mathbf{R}^{N}$. Hence,

$$
\begin{aligned}
H^{*}\left(\omega_{0}\right) & \leq t^{-1}\left[v_{0}(\alpha-t \omega)-v_{0}\left(\alpha-t \omega_{0}\right)\right]+H^{*}(\omega) \\
& \leq R\left|\omega-\omega_{0}\right|+H^{*}(\omega) \quad \forall \omega \in \mathbf{R}^{N},
\end{aligned}
$$

where $R:=\operatorname{Lip}\left(v_{0}\right)$. Now, define

$$
h(q):= \begin{cases}+\infty & \text { if }|q|>R, \\ \left\langle\omega_{0}, q\right\rangle & \text { if }|q| \leq R .\end{cases}
$$

Then $h$ is a lower semicontinuous proper convex function on $\mathbf{R}^{N}$, with $h^{*}(\omega) \equiv R\left|\omega-\omega_{0}\right|$. So (25) implies

$$
H(q) \geq\left\langle\omega_{0}, q\right\rangle-H^{*}\left(\omega_{0}\right) \geq\left\langle\omega_{0}, q\right\rangle-\left(h^{*}+H^{*}\right)(\omega)
$$

for all $\omega \in \mathbf{R}^{N}$. Therefore,

$$
H(q) \geq\left\langle\omega_{0}, q\right\rangle+\sup _{\omega \in \mathbf{R}^{N}}\left\{-\left(h^{*}+H^{*}\right)(\omega)\right\}=\left\langle\omega_{0}, q\right\rangle+\left(h^{*}+H^{*}\right)^{*}(0)
$$

for any $q \in \mathbf{R}^{N}$. Next, consider the "infimum convolute" $h \square H$ given by the formula

$$
(h \square H)(q):=\inf _{q+\bar{q}=q}\{h(\underline{q})+H(\bar{q})\} \equiv \min _{|\underline{q}| \leq R}\left\{\left\langle\omega_{0}, \underline{q}\right\rangle+H(q-\underline{q})\right\} .
$$

This infimum convolute [6, Theorem 16.4] is a (finite) convex function with $(h \square H)^{*}=h^{*}+$ $H^{*}$. It follows from (26) that

$$
H(q) \geq\left\langle\omega_{0}, q\right\rangle+(h \square H)^{* *}(0)=\left\langle\omega_{0}, q\right\rangle+(h \square H)(0),
$$

i.e. that

$$
H(q) \geq\left\langle\omega_{0}, q\right\rangle+\min _{|\bar{q}| \leq R}\left\{H(\bar{q})-\left\langle\omega_{0}, \bar{q}\right\rangle\right\},
$$

for all $q \in \mathbf{R}^{N}$. Thus, there exists a $\bar{q} \in \mathbf{R}^{N},|\bar{q}| \leq R$, such that

$$
H(q) \geq H(\bar{q})+\left\langle\omega_{0}, q-\bar{q}\right\rangle \quad \forall q \in \mathbf{R}^{N} .
$$

Finally, assume, contrary to our claim, that $\left|\omega_{0}\right|>L$. Then, for any fixed $\varepsilon$ with $0<\varepsilon<$ $(r-R) /\left|\omega_{0}\right|$, take $q:=\bar{q}+\varepsilon \omega_{0}$. Of course, $0<|q-\bar{q}|=\varepsilon\left|\omega_{0}\right|<r-R$. Hence $|q|,|\bar{q}|<r$. But, by (27), this $q$ would satisfy

$$
H(q)-H(\bar{q}) \geq\left|\omega_{0}\right| \cdot|q-\bar{q}|>L|q-\bar{q}|,
$$

which contradicts the assumption of the lemma. The proof is thereby completed.

Proof of LemMA 2. For any temporarily fixed $z^{\prime} \in D_{1}, t \geq 0, x^{\prime} \in \mathbf{R}^{n_{1}}$, let

$$
H=H\left(p^{\prime \prime}\right):=-f^{* 1}\left(z^{\prime}, p^{\prime \prime}\right) \quad \text { and } \quad v_{0}=v_{0}\left(x^{\prime \prime}\right):=\phi\left(x^{\prime}-t z^{\prime}, x^{\prime \prime}\right) \text {. }
$$

Obviously, $H$ is a (finite) convex function on $\mathbf{R}^{n_{2}}$, with $H^{*}\left(z^{\prime \prime}\right) \equiv \underline{f}^{*}\left(z^{\prime}, z^{\prime \prime}\right)$ (in view of (10)). For definiteness, suppose that

$$
\left|f\left(p^{\prime}, p^{\prime \prime}\right)-f\left(p^{\prime}, \bar{p}^{\prime \prime}\right)\right| \leq L\left|p^{\prime \prime}-\bar{p}^{\prime \prime}\right| \quad \forall p^{\prime} \in \mathbf{R}^{n_{1}} ; p^{\prime \prime}, \bar{p}^{\prime \prime} \in \mathbf{R}^{n_{2}},\left|p^{\prime \prime}\right|,\left|\bar{p}^{\prime \prime}\right| \leq r
$$


for some $L \geq 0, r>\operatorname{Lip}(\phi)\left(\geq \operatorname{Lip}\left(v_{0}\right)\right)$. Then it can be shown that

$$
\left|H\left(p^{\prime \prime}\right)-H\left(\bar{p}^{\prime \prime}\right)\right| \leq L\left|p^{\prime \prime}-\bar{p}^{\prime \prime}\right| \quad \forall p^{\prime \prime}, \bar{p}^{\prime \prime} \in \mathbf{R}^{n_{2}},\left|p^{\prime \prime}\right|,\left|\bar{p}^{\prime \prime}\right| \leq r .
$$

In fact, given arbitrary $\varepsilon \in(0,+\infty)$ and $p^{\prime \prime}, \bar{p}^{\prime \prime} \in \mathbf{R}^{n_{2}}$, with $\left|p^{\prime \prime}\right|,\left|\bar{p}^{\prime \prime}\right| \leq r$, since $z^{\prime} \in D_{1}$, we could find (using (6) and (18)) a $p^{\prime} \in \mathbf{R}^{n_{1}}$ such that

$$
+\infty>f^{*_{1}}\left(z^{\prime}, \bar{p}^{\prime \prime}\right)>\left\langle z^{\prime}, p^{\prime}\right\rangle-f\left(p^{\prime}, \bar{p}^{\prime \prime}\right)-\varepsilon .
$$

So, (6) together with (28) implies

$$
\begin{aligned}
f^{*_{1}}\left(z^{\prime}, p^{\prime \prime}\right)-f^{*_{1}}\left(z^{\prime}, \bar{p}^{\prime \prime}\right) & \leq f^{*_{1}}\left(z^{\prime}, p^{\prime \prime}\right)-\left\langle z^{\prime}, p^{\prime}\right\rangle+f\left(p^{\prime}, \bar{p}^{\prime \prime}\right)+\varepsilon \\
& \leq\left\langle z^{\prime}, p^{\prime}\right\rangle-f\left(p^{\prime}, p^{\prime \prime}\right)-\left\langle z^{\prime}, p^{\prime}\right\rangle+f\left(p^{\prime}, \bar{p}^{\prime \prime}\right)+\varepsilon \\
& \leq\left|f\left(p^{\prime}, p^{\prime \prime}\right)-f\left(p^{\prime}, \bar{p}^{\prime \prime}\right)\right|+\varepsilon \leq L\left|p^{\prime \prime}-\bar{p}^{\prime \prime}\right|+\varepsilon .
\end{aligned}
$$

Because $\varepsilon \in(0,+\infty)$ is arbitrarily chosen, we get

$$
H\left(\bar{p}^{\prime \prime}\right)-H\left(p^{\prime \prime}\right)=f^{*_{1}}\left(z^{\prime}, p^{\prime \prime}\right)-f^{*_{1}}\left(z^{\prime}, \bar{p}^{\prime \prime}\right) \leq L\left|p^{\prime \prime}-\bar{p}^{\prime \prime}\right|
$$

Similarly,

$$
H\left(p^{\prime \prime}\right)-H\left(\bar{p}^{\prime \prime}\right) \leq L\left|\bar{p}^{\prime \prime}-p^{\prime \prime}\right|=L\left|p^{\prime \prime}-\bar{p}^{\prime \prime}\right| .
$$

Thus (29) has been proved. Therefore, we may apply Lemma 3 to these $H$ and $v_{0}$. (Here, $N:=n_{2}$, while $x^{\prime \prime}$ and $z^{\prime \prime}$ stand for $\alpha$ and $\omega$, respectively.) It follows that (for an arbitrary $\boldsymbol{x}^{\prime \prime} \in \mathbf{R}^{n_{2}}$ )

$$
\begin{aligned}
& \min _{z^{\prime \prime} \in \mathbf{R}^{n_{2}}}\left\{\phi\left(x^{\prime}-t z^{\prime}, x^{\prime \prime}-t z^{\prime \prime}\right)+t \cdot \underline{f}^{*}\left(z^{\prime}, z^{\prime \prime}\right)\right\} \\
& \quad=\min _{z^{\prime \prime} \in \mathbf{R}^{n_{2}}}\left\{v_{0}\left(x^{\prime \prime}-t z^{\prime \prime}\right)+t \cdot H^{*}\left(z^{\prime \prime}\right)\right\}=\min _{\left|z^{\prime \prime}\right| \leq L}\left\{v_{0}\left(x^{\prime \prime}-t z^{\prime \prime}\right)+t \cdot H^{*}\left(z^{\prime \prime}\right)\right\} \\
& =\min _{\left|z^{\prime \prime}\right| \leq L}\left\{\phi\left(x^{\prime}-t z^{\prime}, x^{\prime \prime}-t z^{\prime \prime}\right)+t \cdot \underline{f}^{*}\left(z^{\prime}, z^{\prime \prime}\right)\right\} .
\end{aligned}
$$

Hence, (20) gives us

$$
u_{-}(t, x)=\sup _{z^{\prime} \in D_{1}} \min _{\left|z^{\prime \prime}\right| \leq L}\left\{\phi(x-t z)+t \cdot \underline{f}^{*}(z)\right\}
$$

for any $(t, x) \in \overline{\mathcal{D}}$. Dually in the other case corresponding to (21).

As an immediate consequence of Lemma 2, we have:

COROLlaRY 3. Let $\phi$ be (globally) Lipschitz continuous, $f\left(p^{\prime}, p^{\prime \prime}\right) \equiv H_{1}\left(p^{\prime}\right)+$ $H_{2}\left(p^{\prime \prime}\right)$, with $H_{1}$ concave, $H_{2}$ convex (both finite). Let $L_{1}, L_{2} \geq 0$ be such that, for some $r>\operatorname{Lip}(\phi)$,

$$
\begin{gathered}
\left|H_{1}\left(p^{\prime}\right)-H_{1}\left(\bar{p}^{\prime}\right)\right| \leq L_{1}\left|p^{\prime}-\bar{p}^{\prime}\right| \quad \forall p^{\prime}, \bar{p}^{\prime} \in \mathbf{R}^{n_{1}},\left|p^{\prime}\right|,\left|\bar{p}^{\prime}\right| \leq r, \\
\left|H_{2}\left(p^{\prime \prime}\right)-H_{2}\left(\bar{p}^{\prime \prime}\right)\right| \leq L_{2}\left|p^{\prime \prime}-\bar{p}^{\prime \prime}\right| \quad \forall p^{\prime \prime}, \bar{p}^{\prime \prime} \in \mathbf{R}^{n_{2}},\left|p^{\prime \prime}\right|,\left|\bar{p}^{\prime \prime}\right| \leq r .
\end{gathered}
$$


Then the unique viscosity solution $u \in U C_{x}\left([0,+\infty) \times \mathbf{R}^{n}\right)$ of the Cauchy problem (14)-(15) satisfies on $\overline{\mathcal{D}}$ the inequalities

$$
\begin{array}{r}
\max _{\left|z^{\prime}\right| \leq L_{1}} \min _{\left|z^{\prime \prime}\right| \leq L_{2}}\left\{\phi(x-t z)+t \cdot\left(H_{1}^{*}\left(z^{\prime}\right)+H_{2}^{*}\left(z^{\prime \prime}\right)\right)\right\} \leq u(t, x) \\
\leq \min _{\left|z^{\prime \prime}\right| \leq L_{2}} \max _{\left|z^{\prime}\right| \leq L_{1}}\left\{\phi(x-t z)+t \cdot\left(H_{1}^{*}\left(z^{\prime}\right)+H_{2}^{*}\left(z^{\prime \prime}\right)\right)\right\}
\end{array}
$$

REMARK 5. The strict inequality in the hypothesis " $r>\operatorname{Lip}(\phi)$ " of Lemma 2 and Corollary 3 (or that in " $r>\operatorname{Lip}\left(v_{0}\right)$ " of Lemma 3 ) is essential for the proofs. In this connection, Corollary 3 corrects a result by Bardi and Faggian (cf. [1, Lemma 3.3]), where they take $r:=\operatorname{Lip}(\phi)$, but this is impossible, as the following example shows.

EXAMPLE 1. Let $n_{1}:=1=: n_{2}, \phi \equiv 0, H_{1} \equiv-\left(p^{\prime}\right)^{2} / 2$, and $H_{2} \equiv p^{\prime \prime}$. In this case, $\operatorname{Lip}(\phi)=0$. Then any $L_{1}, L_{2}>0$ surely satisfy all the hypotheses of [1, Lemma 3.3], but the desired estimates become

$$
\begin{gathered}
\max _{\left|z^{\prime}\right| \leq L_{1}} \min _{\left|z^{\prime \prime}\right| \leq L_{2}}\left\{t \cdot\left(\delta\left(z^{\prime \prime} \mid 1\right)-\left(z^{\prime}\right)^{2} / 2\right)\right\} \leq u(t, x) \\
\leq \min _{\left|z^{\prime \prime}\right| \leq L_{2}} \max _{\left|z^{\prime}\right| \leq L_{1}}\left\{t \cdot\left(\delta\left(z^{\prime \prime} \mid 1\right)-\left(z^{\prime}\right)^{2} / 2\right)\right\}
\end{gathered}
$$

that would not be true if $t>0$ and $L_{2}<1$.

REMARK 6. "Duality" of the results in this section can be established for the case where $f$ is merely continuous, but $\phi=\phi\left(x^{\prime}, x^{\prime \prime}\right)$ is concave-convex and (globally) Lipschitz continuous, as is done in [1] when $\phi\left(x^{\prime}, x^{\prime \prime}\right) \equiv u_{1}\left(x^{\prime}\right)+u_{2}\left(x^{\prime \prime}\right)$, with $u_{1}$ concave, $u_{2}$ convex (both Lipschitz continuous).

EXAMPLE 2. Let $n_{1}=n_{2}:=k>0$, and $f_{1}(p):=\left\langle p^{\prime}, p^{\prime \prime}\right\rangle$ for $p=\left(p^{\prime}, p^{\prime \prime}\right) \in$ $\mathbf{R}^{k} \times \mathbf{R}^{k}$. Then $f_{1}$ is trivially a concave-convex function. (One can also check directly that it is neither convex nor concave.) There could not be any functions $g_{1}=g_{1}\left(p^{\prime}\right), g_{2}=g_{2}\left(p^{\prime \prime}\right)$, with $f_{1}(p) \equiv g_{1}\left(p^{\prime}\right)+g_{2}\left(p^{\prime \prime}\right)$. To find a (finite) concave-convex function $f$ on $\mathbf{R}^{n_{1}} \times \mathbf{R}^{n_{2}}$ with (3)-(4) holding, for which there do not exist any (concave) $H_{1}=H_{1}\left(p^{\prime}\right)$ and (convex) $H_{2}=H_{2}\left(p^{\prime \prime}\right)$ such that $f(p) \equiv H_{1}\left(p^{\prime}\right)+H_{2}\left(p^{\prime \prime}\right)$, we can now take, for example,

$$
f(p):=-\left|p^{\prime}\right|^{\sqrt{2}}+\left\langle p^{\prime}, p^{\prime \prime}\right\rangle+\left|p^{\prime \prime}\right|^{3 / 2} \text { for } p=\left(p^{\prime}, p^{\prime \prime}\right) \in \mathbf{R}^{n_{1}} \times \mathbf{R}^{n_{2}} \text {. }
$$

Acknowledgement. The author wishes to thank Professors M. Tsuji and M. Bardi for several stimulating discussions.

\section{References}

[ 1 ] M. BARDI and S. FAGGIAN, Hopf-type estimates and formulas for non-convex non-concave Hamilton-Jacobi equations, SIAM J. Math. Anal. 29 (1998), 1067-1086.

[ 2 ] M. G. CRANDALL and P. L. LiONS, On existence and uniqueness of solutions of Hamilton-Jacobi equations, Nonlinear Anal. 10 (1986), 353-370.

[ 3 ] E. HOPF, Generalized solutions of nonlinear equations of first-order, J. Math. Mech. 14 (1965), 951-973. 
[ 4 ] H. IsHII, Uniqueness of unbounded viscosity solution of Hamilton-Jacobi equations, Indiana Univ. Math. J. 33 (1984), 721-748.

[ 5 ] - Perron's method for Hamilton-Jacobi equations, Duke Math. J. 55 (1987), 369-384.

[ 6 ] R. T. RockAfellar, Convex analysis, Princeton University Press (1970).

[ 7 ] T. D. VAN, M. TSUJi and N. D. Thai SON, The characteristic method and its generalizations for firstorder nonlinear partial differential equations, Chapman \& Hall/CRC Monographs and Surveys in Pure and Applied Mathematics, Vol. 101, Boca Raton (1999).

Present Address:

DePartment of PDEs, hanoi Institute of Mathematics, P.O. BOX 631, Bo-Ho 10000 HANOI, VIETNAM.

e-mail: thaison@thevinh.ncst.ac.vn 